\title{
Piroxicam Loaded Solid Lipid Nanoparticles (SLNs): Potential for Topical Delivery
}

\author{
Harshita Krishnatreyya ${ }^{1, \star}$, Sanjay Dey ${ }^{2}$, Paulami Pal', Pranab Jyoti Das ${ }^{1}$, Vipin Kumar Sharma ${ }^{3}$, \\ Bhaskar Mazumder ${ }^{1}$
}

${ }^{1}$ Department of Pharmaceutical Sciences, Dibrugarh University, Dibrugarh, Assam, INDIA.

${ }^{2}$ Department of Pharmaceutics, School of Pharmacy, Techno India University, West Bengal, INDIA.

${ }^{3}$ Department of Pharmaceutical Sciences, Gurukul Kangri University, Haridwar, Uttarakhand, INDIA.

\begin{abstract}
Aim: The objective of this study was to develop suitable lipid nanocarriers for topical delivery of Piroxicam, known for its anti-inflammatory and anti-arthritic properties and to increase its therapeutic potential and residence time at the site of inflammation and in systemic circulation. Materials and Methods: Piroxicam loaded Solid lipid nanoparticles (SLNs) were prepared by high-speed homogenization followed by ultrasonication technique. Physicochemical evaluation of SLNs involved particle size and zeta potential analysis, electron micrographic analysis, drug loading, entrapment and release studies. Drug excipient interactions were studied by Fourier transform infrared spectroscopy (FTIR) and Differential scanning calorimetry (DSC). Prepared nanoparticles were assimilated into a topical gel system and evaluated for viscosity, in vitro permeation properties and in vivo efficacy assessment by carrageenan induced rat paw edema study. Results: Evaluation of prepared SLNs revealed the particle size, drug loading, entrapment efficiency and in vitro release to significantly differ $(p<0.05)$ with different lipid and surfactant concentrations present in formulations. SEM and TEM were useful aids in studying the morphology of the SLNs. FTIR and DSC revealed no likely interaction amongst the drug and/or any excipients. In vitro permeation and in vivo anti-inflammatory study of the SLN gel revealed it to be a sustained permeation system, maintaining drug concentration over an extended period of time and providing a better therapeutic potential on topical dermal application when compared with a free drug gel. Conclusion: Our results proved the suitability and capability of fabricated piroxicam loaded SLN gel system in treating arthritic pain and inflammation when delivered topically.
\end{abstract}

Key words: Solid lipid nanoparticles, Piroxicam, Topical, Inflammation, Dermal.

\section{INTRODUCTION}

Piroxicam belongs to the oxicam class of Non-steroidal anti-inflammatory drug (NSAID) used in the treatment of rheumatoid arthritis, osteoarthritis, alkalosing spandylitis and acute gout. ${ }^{1,2}$ It is proposed as a Biopharmaceutical Classification System (BCS) class II drug with low solubility ( $\mathrm{pKa}$ 6.3) and high permeability. ${ }^{3}$ Administration of piroxicam by traditional methods (orally or intramuscularly) resulted in severe side effects and toxicity like nausea, vomiting, dyspepsia, epigastric pain, gastrointestinal ulceration and higher risk of renal failure or bleeding., ${ }^{4,5}$ As opposed to the traditional methods, transdermal and/ or topical drug delivery system possesses several advantages such as protection from gastrointestinal enzymatic degradation, bypass of hepatic first-pass metabolism and lower risk of systemic side effects. ${ }^{6}$ Therefore, this study was designed so as to obtain a safe and effective therapeutic outcome by developing a suitable carrier for the topical delivery of piroxicam. The main obstacle of delivery of drug through topical route is the permeation of drug through skin stratum corneum. There are several strategies such as the use of vesicular formulation as a
Submission Date: 13-09-2018; Revision Date: 28-12-2018; Accepted Date: 20-03-2019

DOI: 10.5530/ijper.53.2s.52 Correspondence: Ms. Harshita Krishnatreyya Department of Pharmaceutical Sciences Dibrugarh University Dibrugarh, Assam786004, INDIA.

Phone: +91 8638095195

E-mail: harshita.

krishnatreyya@gmail.com

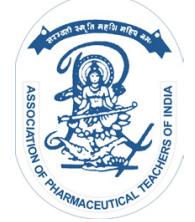

www.ijper.org 
delivery vehicle, use of penetration enhancers (Chemical approach) and physical methods (Like: iontophoresis, sonophoresis, etc.) have been reported earlier. ${ }^{7-13}$ Unfortunately, the physical and chemical methods are expensive and may cause skin irritation.

Nanoparticles composed of lipid materials such as wax or fats seem attractive carrier system for the transdermal delivery of drug. ${ }^{14,15}$ They have several advantages over traditional method of transdermal drug delivery. Nanoparticles made of solid lipid display unique benefits in drug delivery and produce various desirable effects. They moisten the skin through occlusion by providing skin hydration. ${ }^{16}$ Solid lipid nanoparticles (SLNs) are suitable for use as damaged or inflamed skin as they are made up of physiological and biodegradable lipids that are non-irritant and non-toxic. Several studies reported the favourable incorporation of drugs into SLN and its advantages as a colloidal carrier system. ${ }^{17-19}$ In the present study, SLN was selected as delivery carrier for delivering piroxicam through topical route. The present work was designed mainly with the aim of formulating, evaluating and consequently screening piroxicam incorporated SLNs for the possibility of any anti-inflammatory efficacy when applied by the topical route of drug delivery.

\section{MATERIALS AND METHODS}

Piroxicam was procured as a gift sample from Alkem Laboratories, Sikkim, India.

Phospholipon 80 was obtained as gift sample from Lipoid, Ludwigshafen, Germany. Stearic acid and Pluronic F68 were bought from Himedia Chemicals, Mumbai, India. All reagents and chemicals used in this study were of analytical grade and used as received without additional purification.

\section{Fabrication of Piroxicam loaded SLNs}

Piroxicam loaded SLNs were developed by high-speed homogenization followed by ultrasonication technique. The composition of piroxicam loaded SLNs is presented in Table 1. In brief, accurate weighed amounts of piroxicam and surfactants were dissolved in $10 \mathrm{~mL}$ of methanol. On the other hand, exact amount of lipid was taken in $10 \mathrm{ml}$ of acetone and stirred for 5 min. Both the mixtures were maintained at the same temperature $\left(60^{\circ} \mathrm{C}\right)$. After thermal treatment, both of these were admixed. The mixture was then sonicated for 15 min using a probe sonicator (Ultrasonic Processor, Hielscher, Germany). This solution constituted the organic phase. In another beaker, weighed quantity of surfactant was dissolved in $25 \mathrm{~mL}$ of distilled water and heated at $60^{\circ} \mathrm{C}$. This solution constituted the aqueous phase. Both organic and aqueous phases were mixed together and homogenized by high-speed homogenizer (Ultra Turrax ${ }^{\circledR}$ T 25, Janke and Kunkle GmbH, Germany) at $15000 \mathrm{rpm}$ for $8 \mathrm{~min}$. The homogenized solution was sonicated for 20 min to prevent agglomeration of smaller-size particles. The obtained formulation was stirred using magnetic stirrer (Remi, Mumbai, India) for $30 \mathrm{~min}$, filtered through $0.2 \mu \mathrm{m}$ Whatman filter paper and centrifuged at 20,000 rpm for $20 \mathrm{~min}$ (Remi Instruments, India). The residue was collected while discarding the supernatant and collected sediment was further lyophilized to obtain its free-flowing powder form.

\section{Characterization of SLNs}

\section{Particle size and zeta potential analysis}

Particle-size analysis of piroxicam SLNs was performed by dynamic light scattering (DLS) method using a Malvern Zetasizer Nano ZS (Malvern Instruments, UK). DLS yielded mean particle size and polydispersity index (PDI), which measures the width of size dispersion. The particle size and PDI of the samples were investigated by calculating average of 10 measurements at an angle of $90^{\circ} \mathrm{C}$ in $10 \mathrm{~mm}$ diameter disposable plastic cell at $25^{\circ} \mathrm{C}$. Before measurement, the samples were diluted in distilled water to yield a suitable scattering intensity. Zeta potential determination of piroxicam SLNs was done by the same Malvern Zetasizer Nano ZS (Malvern Instruments, UK). The measurements were carried out in distilled water adapted to $50 \mathrm{mS} / \mathrm{cm}$ conductivity with sodium chloride solution $(0.9 \% \mathrm{w} / \mathrm{v})$ and field strength was applied $20 \mathrm{~V} / \mathrm{cm}$. During measurement the $\mathrm{pH}$ range was $5.5-6.0$. Instrumental zeta potential was determined using the Helmholtz-Smoulchowsky equation.

\section{Electron microscopic analysis}

Both Scanning electron microscopy (SEM) and Transmission electron microscopy (TEM) were done to directly observe the piroxicam loaded SLNs. These techniques provide particle specifics such as surface topography and external morphology of the samples. TEM has a small size limit of detection as compared to SEM. Samples were sent to the North Eastern Hill University, Shillong, India for TEM while SEM analysis of the samples were done in Oil India Limited, Duliajan, Assam, India.

\section{Drug loading and entrapment efficiency}

Drug loading and entrapment efficiency of piroxicam SLNs were adjudged by the centrifugation technique. ${ }^{20}$ $1 \mathrm{ml}$ of centrifuged SLN sediment (As mentioned in the SLN preparation method) was taken and diluted with acetonitrile. The diluted sample was analysed by UV-Visible spectrophotometer (UV-1700, Shimadzu, Tokyo, Japan) at $\lambda_{\max }$ of $230 \mathrm{~nm}$. The drug loading and 
entrapment efficiency of piroxicam in SLNs were calculated according to following equations:

determined

Drug loading $(\%)=\frac{\text { Drug content determined }}{\text { Drug content vailable }}+$ weight of lipid $\times 100$
Entrapment efficiency $(\%)=\frac{\text { Drug content }}{\text { Amount of drug initially added }} \times 100$

\section{In vitro Drug Release Study}

In vitro release study of piroxicam SLNs was studied using the Franz diffusion apparatus method. Typically, 1 $\mathrm{mL}$ of the centrifuged SLN sediment (As mentioned in the SLN preparation method) was placed on the dialysis membrane having a molecular weight cutoff: 10,000 -12,000 (HiMedia, Mumbai, India). The membrane was adjusted between the donor and receptor sections of the diffusion apparatus which was then filled with $200 \mathrm{~mL}$ phosphate buffer, $\mathrm{pH}$ 5.8, which served as the release medium. The release medium was stirred at 175 rpm in a magnetic stirrer (Remi, Mumbai, India) and the temperature was kept at $37 \pm 0.5^{\circ} \mathrm{C}$ during the experimental procedure. At specific time intervals, $1 \mathrm{~mL}$ of aliquot was withdrawn and reinstated with equal volume of fresh buffer to preserve the sink condition. Aliquots were diluted suitably with acetonitrile and the quantity of piroxicam contained in the samples was evaluated using a UV-Visible spectrophotometer (UV-1700, Shimadzu, Tokyo, Japan) at? $\max$ of $230 \mathrm{~nm}$. The release of individual SLN formulations was done in triplicate.

\section{Drug Release Kinetics}

The in vitro drug release pattern from piroxicam SLNs was predicted by applying the drug release data to different release kinetic models like: zero-order, first-order, Higuchi, Korsmeyer-Peppas and Hixson-Crowell. For each release kinetics model, the values of $R^{2}$ (Correlation coefficient) and $\mathrm{n}$ (Release exponent of KorsmeyerPeppas kinetics), K (Rate constant) and SSR (Sum of squared residual) were determined for each formulation and all the models and the possible methods of drug release were identified.

\section{Optimization of Suitable SLN Formulation}

Suitable formulation from amongst the prepared piroxicam SLN formulations was selected, based on their optimum particle size and zeta potential, high drug loading, entrapment efficiency and better in vitro drug release properties. The optimized formulation was further scrutinized and used for additional studies.

\section{Incorporation of Piroxicam-loaded SLN into Gel System}

Gel containing appropriate formulation of piroxicam SLN and pure piroxicam were made by using $0.5 \% \mathrm{w} / \mathrm{v}$ of carbopol 934P as gel forming polymer. Firstly, gel was prepared by diffusing carbopol 934P in distilled water containing glycerol (10\%) and kept for saturation for $3 \mathrm{~h}$. Lyophilized piroxicam-loaded SLN was admixed with aqueous carbopl 934P dispersion under controlled stirring using a magnetic stirrer (Remi, Mumbai, India) to obtain uniform and smooth dispersion containing a final concentration of $20 \% \mathrm{w} / \mathrm{w}$ piroxicam-loaded SLN. The $\mathrm{pH}$ of the piroxicam-loaded SLN enriched gel was adjusted to 6.0 using sufficient quantity of triethanolamine. Piroxicam conventional gel was prepared in similar process and was used as reference during efficacy evaluation.

\section{Evaluation of Piroxicam SLN gel system}

\section{Appearance}

Organoleptic properties like color, odor, phase separation and gel capacity to be washed out were visually observed in the SLN gel formulation.

\section{Physicochemical properties}

$\mathrm{pH}$ of piroxicam SLN gel was evaluated using a digital pH meter (Model-355, Systronic, India). The viscosity and rheological properties of the water based piroxicam SLN gels as compared to prepared conventional gel was determined using a Brookefield viscometer (Brookfield Engineering Laboratories, USA) with spindle no. 6 at 10 rpm. All measurements were performed at a temperature of $37 \pm 0.5^{\circ} \mathrm{C}$.

\section{Skin Irritation}

A small quantity of the prepared SLN gel was applied on the skin and evaluated for its easy removal after 1 min. This was done to observe the presence of rough or granular surface particles and examine the skin for any sign/symptoms of irritation.

\section{Drug-excipient interaction study}

\section{Fourier transform infrared spectra (FTIR) spectroscopy}

FTIR spectra was evaluated to check the compatibility of the drug with formulation excipients and to ensure proper entrapment of drug in formulation. The FTIR spectra was recorded using an FTIR spectrophotometer (Shimadzu, Tokyo, Japan; Model: 8400) in between the range of $600-4000 \mathrm{~cm}^{-1}$ using $\mathrm{KBr}$ discs to recognize the variations in the characteristic peaks of individual drug piroxicam and piroxicam loaded SLN formulations. Pure drug was pulverized with dried potassium bromide. This mixture was then pressed in a hydraulic press to form a transparent pellet and the pellet FTIR spectrum was measured. To observe the spectra of the piroxicam SLN gel, a drop of sample (Around $5-50 \mu \mathrm{L})$ was spread on the plate and the IR spectrum was recorded. Distinctive functional groups present in 
each sample was identified and compared to check for specific drug excipient interactions.

\section{Differential Scanning Calorimetry (DSC)}

Thermal behaviour of pure piroxicam drug and piroxicam-loaded SLNs was analysed using a differential scanning calorimeter (Perkin Elmer, USA). Approximately $10 \mathrm{mg}$ of sample was placed in aluminium crimp cells and heated at the scanning rate of $10^{\circ} \mathrm{C} / \mathrm{min}$ from 30 to $400^{\circ} \mathrm{C}$ in a nitrogen atmosphere. Aluminium oxide was used as the reference material to calibrate the temperature and energy scale of the DSC instrument.

\section{In vitro Permeation Study}

The in vitro permeation study of piroxicam SLN gels was done using pig ear epidermal skin obtained from the local slaughter house. The subcutaneous fat on the skin was removed and soaked in $2 \mathrm{M}$ sodium bromide solution for $36 \mathrm{~h}$. The pig ear epidermis was peeled from the dermis, washed with double distilled water and used for further study. The isolated epidermis was mounted carefully across the donor and receptor sections in Franz Diffusion cell with the epidermal side up and an effective diffusion area of $3.8 \mathrm{~cm} .{ }^{2}$ The receptor section remained filled with $50 \mathrm{~mL}$ of phosphate buffer (pH 5.8) and was maintained at $37 \pm 0.5^{\circ} \mathrm{C}$ with stirring at $600 \mathrm{rpm}$. The skin membranes were calibrated for 30 min before placing gel into donor compartment. Then $1 \mathrm{~g}$ of piroxicam SLN gel and conventional gel were placed individually in the donor section of the diffusion cell. Sampling was done at predetermined intervals. At specific points, $2.5 \mathrm{ml}$ aliquots were withdrawn from the receptor section and immediately an equal volume of receptor fluid was replaced in it. Piroxicam concentration in withdrawn sample was analysed spectrophotometrically using a UV-Visible spectrophotometer at max $260 \mathrm{~nm}$. The permeation of both gels were carried out in triplicate.

\section{Permeation Data Analysis}

The permeation profile of piroxicam from piroxicam loaded SLN gel and conventional gel was made by plotting the cumulative amount of piroxicam permeated per unit pig skin epidermis area $\left(\mu \mathrm{g} / \mathrm{cm}^{2}\right)$ versus time. The steady state flux $\left(\mathrm{Jss}, \mu \mathrm{g} / \mathrm{cm}^{2} / \mathrm{h}\right)$ of piroxicam was determined from the slope of graph by applying linear regression analysis. The permeability coefficient $(\mathrm{Kp})$ of piroxicam was calculated using following equation:

$$
\mathrm{K}_{\mathrm{p}}=\frac{\mathrm{J}_{s s}}{\mathrm{C}}
$$

Where, Jss is the steady state flux $\left(\mu \mathrm{g} / \mathrm{cm}^{2} / \mathrm{h}\right)$ of piroxicam from the gel and $\mathrm{C}$ is the initial piroxicam concentration available in donor section. The enhancement ratio (Er) of piroxicam SLN gel was determined by following expression:

$E_{r}=$ Jss of piroxicam from SLN gel /Jss of piroxicam from conventional gel

\section{In vivo Anti-Inflammatory Efficacy of Piroxicam SLN Gel}

\section{Ethical statement for the use of experimental animals}

Wistar rats (Rattus norvegicus) were used as experimental animals. All animal experiments complied with the ARRIVE guidelines ${ }^{12}$ and were carried out in accordance with the U.K. Animals (Scientific Procedures) Act 1986 and associated guidelines. They were used as per the guidelines of the Institutional Animal Ethics Committee of the Department of Pharmaceutical Sciences, Dibrugarh University, Assam, India (Regd No. 1576/ GO/a/11/CPCSEA, Dated: 17/02/2012) under the approval number: IAEC/DU/95. All applicable international, national and/or institutional guidelines for the care and use of animals were followed. The in vivo anti-inflammatory potential of formulated piroxicam SLN gel was evaluated by the carrageenen induced paw edema method using Wistar albino rats as the experimental animals. Animal care and handling throughout the experimental procedure were carried out in accordance with the CPSEA guidelines.

The rats were acclimatized at the commencement of the study and were divided into four groups $(n=6)$ as follows: Group 1: Normal saline (control) Group 2: Carrageenan and normal saline (toxicant) Group 3: Piroxicam conventional gel (standard) Group 4: Piroxicam loaded SLN gel (test) For the paw edema study, a digital plethysmometer was used to measure the volume of the rat paw (in $\mathrm{ml}$ ). The plethysmometer was designed so as to measure small changes in volume. The rats were marked on the left hind paw at a specific position. Every now and then, the paw was dipped to that fixed mark in an electrolyte fluid so that every time the paw is dipped in the electrolyte fluid, it would be dipped to that fixed mark which would assure constant paw volume. The original paw volume of the rats were measured and noted. Paw edema was induced in the rats using freshly prepared $1 \%$ carrageenan saline solution. $0.1 \mathrm{ml}$ of prepared carrageenen solution was injected into the plantar region of the left hind paw of the rats. After the induction of edema, the enlarged paw volumes were noted. Prepared free drug gel as well as SLN gel formulation were applied to the third and fourth groups.

Readings of the paw volume were then taken after 1 , $2,4,8,10$ and 24 h of time intervals using the plethysmometer. The percentage edema rate and percentage edema inhibition rate were calculated using following equations:

$\%$ Edema rate $=\mathrm{V}_{\mathrm{t}} \mathrm{V}_{0} / \mathrm{V}_{0} \times 100 \%$ 
$\%$ Edema inhibition rate $=\mathrm{E}_{\mathrm{S}} \mathrm{E}_{\mathrm{t}} / \mathrm{E}_{\mathrm{S}} \times 100$. Here, V0 and $\mathrm{V}_{\mathrm{t}}$ indicate mean paw volume before and after carrageenan injection $(\mathrm{mL})$, respectively, $\mathrm{E}_{\mathrm{S}}$ and $\mathrm{E}_{\mathrm{t}}$ indicate the edema rate of standard group and test group, respectively.

\section{Statistical Analysis}

All data were presented as Mean \pm Standard deviation (S.D). Statistical analysis was carried out by one way analysis of variance (ANOVA) by utilizing the two-tailed paired ' $\mathrm{t}$ ' test (GraphPad InStat Demo Version). $P$ values $<0.05$ were considered to be statistically significant.

\section{RESULTS AND DISCUSSION}

\section{Fabrication of Piroxicam SLNs}

Formulation of piroxicam SLNs was done by highspeed homogenization and ultrasonication technique. Formulation compositions are presented in Table 1. In particular, application of homogenization method resulted in the formation of unstable dispersion with broad dimensional distribution. Conversely, application of homogenization followed by sonication resulted in a stable and homogenous formulation which was free from aggregation. Stearic acid was used as lipid matrix for the fabrication of piroxicam SLNs. Pluronic F68 and phospholipon 80 were used as surfactant and cosurfactant respectively. Varied proportions of the lipid matrix and pluronic F68 were utilized to constitute the SLNs. The fabricated piroxicam loaded SLNs were subjected to different characterization.

\section{Characterization of Piroxicam Loaded SLN}

\section{Particle size and zeta potential}

Particle size and zeta potential data of piroxicam SLNs are depicted in Table 2. The particle size of piroxicam SLNs was found in the range of $136.0 \pm 15.6 \mathrm{~nm}$ to $279.1 \pm 21.3 \mathrm{~nm}$. It was clear from Table 2 that the particle size decreased with increasing concentration of Pluronic F68 in piroxicam loaded SLN formulations. The increasing of pluronic F68 content in SLN formulations could decrease the interfacial tension between lipid matrix and the dispersion medium (Aqueous phase), consequently favour the formation of SLNs with smaller particle size. ${ }^{21}$ The particle size was increased upon increasing the amount of stearic acid in the SLN formulations. The polydispersity index (PDI) of piroxicam SLNs was found to be within an acceptable range of $0.206 \pm 0.091$ to $0.479 \pm 0.045$. Lower PDI values of formulations are considered better as they indicate the formation of uniform particles. Optimum concentrations of Pluronic F68 and stearic acid are relevant as the PDI of SLNs were found to increase with increas- ing lipid and surfactant concentrations. Zeta potential is a potential parameter to access the stability of SLN dispersion. ${ }^{22}$ The zeta potential of piroxicam SLNs was found in the range of $-14.5 \pm 2.7 \mathrm{mV}$ to $-27.5 \pm 1.9$ $\mathrm{mV}$. Generally, particles are considered stable when the zeta potential of colloidal dispersed particles above 30 $\mathrm{mV}$ and this is due to electrostatic repulsion between particles. ${ }^{23}$ The zeta potential values of SLNs revealed the stability of the formulated piroxicam SLNs. Values of zeta potential were found to increase with increased Pluronic F68 content in SLNs whereas increased stearic acid content reduced the zeta potential of formulated SLNs.

\section{Electron Microscopy Analysis}

TEM photomicrographs of piroxicam SLNs is presented in Figures 1. The TEM photomicrograph revealed the ideal spherical shape of piroxicam SLNs which were distinct and free of aggregates (Figures 1A, 1B). Magnification of single SLN shows smooth surface and uniform dispersion of piroxicam throughout the lipid matrix (Figures 1A). Figures 2 shows SEM photomicrographs of piroxicam SLNs. Smooth and uniform surface of a nanoparticle (Crushed) can be seen in Figures $2 \mathrm{~A}$ while Figures $2 \mathrm{~B}$ shows the overall formulation with the presence of distinct SLN particles.

\section{Drug Loading and Entrapment Efficiency}

The drug loading and entrapment efficiency data of piroxicam SLNs are shown in Table 2. The drug loading and entrapment efficiency of piroxicam SLN was found in the range of $0.437 \pm 0.08 \%$ to $0.649 \pm 0.09 \%$ and $50.9 \pm 1.77 \%$ to $74.0 \pm 1.35 \%$, respectively. The concentration of Pluronic F68 and stearic acid might have played crucial roles in improving piroxicam incorporation in the SLNs. The entrapment of piroxicam in SLNs was increased when both Pluronic F68 and stearic acid are increased. Pluronic F68 also contributes towards increasing the solubility of Piroxicam within the lipid matrix. The enhanced entrapment efficiency of piroxicam in SLN upon addition of stearic acid could be due increasing the amount of available lipid matrix for incorporation of piroxicam.

\section{In vitro Drug Release and Release Kinetic Study}

The in vitro release profile of piroxicam from different SLNs are shown in Figures 3. The percentage of piroxicam release from formulations varied from $44.6 \pm$ $1.286 \%$ to $81.70 \pm 1.02 \%$ within 24 h of release study. The in vitro release profile revealed a faster release at the initial stage followed by prolonged release. Such a release can be advantageous as it provides the initial therapeutic concentration of the drug which provides faster relief after dermal application. However, the sustained release 
effect as shown in release pattern may provide the drug over a prolonged period of time. The initial faster release can be explained by the enrichment of piroxicam at outer surface of SLN and the prolonged release can be due to slower diffusion of piroxicam from the solid matrix of SLN.

\section{Drug Release Kinetics}

The obtained SLN release data were fitted with the Zero order, First order, Higuchi, Hixson Crowell and Korsmeyer Peppas kinetic drug release models (Table 3). The best fit with the highest regression coefficient was found in case of the Korsmeyer Peppas model while the drug was also found to show zero order model release. Zero order release indicates the release from systems which is not dependent of the amount of substances present. In case of Korsmeyer Peppas models, the diffusion exponent " $n$ " in all the cases has been found to be as follows: $0.5<n<1.0$ where $\mathrm{n}$ is the diffusion exponent; which indicates drug release from the prepared SLN dispersion is found to follow Anomalous (Non Fickian) diffusion mechanism.

\section{Optimization of Piroxicam Loaded SLN Gel System}

On the basis of optimized values obtained from particle size, drug entrapment and release studies, the piroxicam SLN formulation F2 (Containing 1.00\% Stearic acid and $1.50 \%$ Pluronic F-68) was chosen for further study. For topical delivery, the SLNs were lyophilized and incorporated into carbopol 934P gel system.

\section{Evaluation of Piroxicam SLN Gel System}

Appearance: Piroxicam loaded SLN incorporated gel was found to be clear and smooth in texture, translucent and showed homogenous consistency.

\section{Physicochemical parameters}

Optimum $\mathrm{pH}$ and viscosity values of the SLN gel was obtained which was adequate for topical cutaneous administration as shown in Table 4. The viscosity measurement studies of the prepared SLN gel was performed by Brookfield rheometer as shown in Figures 4. The SLN dispersions generally possessed a lipid matrix of $10-20 \%$ and $90-80 \%$ water because of which they were less visco-elastic by nature. The rheological properties of topical formulations influences its spreadability and contact time on the skin surface. The thixotropic nature of the gel (Figures 4) was confirmed from the curve obtained from rheological studies. The obtained curves affirm that the gel is effectively viscous in nature and the effect of shear rate on its viscosity can be observed.

\section{Skin irritation}

The optimized SLN gel formulation produced no signs of skin irritation (Table 4) or toxicity in experimental animals and was hence, considered to be acceptable for topical application.

\section{Drug-Excipient Interaction Study}

\section{FTIR}

The FTIR spectra of pure piroxicam and piroxicam SLN gel is shown in Figures 4. The FTIR analysis exhibited no distinct physical or chemical interaction of the drug (Figures 5A) with either the lipids (Figures 5B, 5D) or surfactant (Figures 5C). The FTIR spectrum of the pure piroxicam (Figures 5A) showed a small peak at $3333.14 \mathrm{~cm}^{-1}$ corresponding to $\mathrm{N}-\mathrm{H}$ and $\mathrm{O}-\mathrm{H}$ stretching vibrations. Major peaks were observed at $1526 \mathrm{~cm}^{-1}$ and $1431 \mathrm{~cm}^{-1}$ due to $\mathrm{N}-\mathrm{O}$ asymmetric stretching. The presence and position of peaks in FTIR spectrum of piroxicam SLN gel (Figures 5D) showed spectral similarity to an extent with peak positions of FTIR spectrum of pure piroxicam. The characteristic peaks of Piroxicam were observed in the SLN gel indicating little interaction while a few peaks (at near about $1500 \mathrm{~cm}^{-1}$ ) were found to be missing which might be an indication of proper entrapment of the drug within the lipid matrix.

\begin{tabular}{|c|c|c|c|c|c|}
\hline \multicolumn{5}{|c|}{ Table 1: Composition of Solid-Lipid Nanoparticles Containing Piroxicam. } \\
\hline Formulation & Drug (\%w/v) & Stearic acid (\%w/v) & Pluronic F68 (\%w/v) & Phospholipon 80 (\%w/v) & Water (\%v/v) \\
\hline F1 & 0.01 & 1.00 & 1.00 & 0.02 & 100 \\
\hline F2 & 0.01 & 1.00 & 1.50 & 0.02 & 100 \\
\hline F3 & 0.01 & 1.00 & 2.00 & 0.02 & 100 \\
\hline F4 & 0.01 & 0.75 & 1.00 & 0.02 & 100 \\
\hline F5 & 0.01 & 1.25 & 1.00 & 0.02 & 100 \\
\hline F6 & 0.01 & 1.25 & 2.00 & 0.02 & 100 \\
\hline F7 & 0.01 & 1.50 & 2.00 & 0.02 & 100 \\
\hline
\end{tabular}




\begin{tabular}{|c|c|c|c|c|c|}
\hline \multicolumn{7}{|c|}{ Table 2: Characteristics of Fabricated Piroxicam Loaded SLNs. } \\
\hline $\begin{array}{c}\text { Formulation } \\
\text { code }\end{array}$ & Particle size (nm)* & PDI* & $\begin{array}{c}\text { Zeta potential } \\
(\mathbf{m V})^{*}\end{array}$ & $\begin{array}{c}\text { Drug loading } \\
(\%)^{*}\end{array}$ & $\begin{array}{c}\text { Entrapment } \\
\text { efficiency }(\%)^{*}\end{array}$ \\
\hline F1 & $268.4 \pm 16.8$ & $0.325 \pm 0.102$ & $-25.2 \pm 2.5$ & $0.481 \pm 0.03$ & $60.2 \pm 2.46$ \\
\hline F2 & $237.0 \pm 21.3$ & $0.285 \pm 0.015$ & $-20.6 \pm 1.0$ & $0.547 \pm 0.07$ & $64.8 \pm 1.32$ \\
\hline F3 & $136.0 \pm 15.6$ & $0.206 \pm 0.091$ & $-19.2 \pm 2.3$ & $0.589 \pm 0.05$ & $69.0 \pm 2.01$ \\
\hline F4 & $242.4 \pm 24.9$ & $0.312 \pm 0.023$ & $-14.5 \pm 2.7$ & $0.437 \pm 0.08$ & $50.9 \pm 1.77$ \\
\hline F5 & $279.1 \pm 28.7$ & $0.479 \pm 0.045$ & $-27.5 \pm 1.9$ & $0.508 \pm 0.04$ & $63.4 \pm 1.69$ \\
\hline F6 & $190.0 \pm 30.1$ & $0.292 \pm 0.082$ & $-24.2 \pm 1.6$ & $0.649 \pm 0.09$ & $74.0 \pm 1.35$ \\
\hline F7 & $302.1 \pm 22.4$ & $0.465 \pm 0.276$ & $-22.1 \pm 1.2$ & $0.542 \pm 0.21$ & $62.3 \pm 2.21$ \\
\hline
\end{tabular}

\section{Table 3: The release data of the SLNs were fitted into different kinetic models to determine the mechanism of} drug release

\begin{tabular}{|c|c|c|c|}
\hline Formulation code & Kinetic model & Equation & $\mathbf{R}^{2}$ \\
\hline \multirow[t]{5}{*}{ F1 } & Zero & $y=0.144 x+0.976$ & 0.9021 \\
\hline & First & $y=0.0133 x+1.292$ & 0.7545 \\
\hline & Higuchi & $y=0.116 x+0.3814$ & 0.8138 \\
\hline & Hixson Crowell & $y=0.234 x+2.232$ & 0.1848 \\
\hline & Korsmeyer Peppas & $y=0.6014 x+0.572, n=0.6014$ & 0.9462 \\
\hline \multirow[t]{5}{*}{ F2 } & Zero & $y=0.6171 x+0.572$ & 0.9123 \\
\hline & First & $y=0.0173 x+1.533$ & 0.2822 \\
\hline & Higuchi & $y=0.1294 x+0.396$ & 0.8469 \\
\hline & Hixson Crowell & $y=0.989 x+0.3324$ & 0.4678 \\
\hline & Korsmeyer Peppas & $y=0.4830 x-0.135, n=0.4830$ & 0.9594 \\
\hline \multirow[t]{5}{*}{ F3 } & Zero & $y=0.1710 x+0.5311$ & 0.7598 \\
\hline & First & $y=0.0182 x+0.1603$ & 0.3808 \\
\hline & Higuchi & $y=0.1306 x+0.358$ & 0.7508 \\
\hline & Hixson Crowell & $y=0.6125 x+0.221$ & 0.5314 \\
\hline & Korsmeyer Peppas & $y=0.074 x-0.1786, n=0.074$ & 0.8854 \\
\hline \multirow[t]{5}{*}{$\mathrm{F} 4$} & Zero & $y=0.1775 x+0.5002$ & 0.9621 \\
\hline & First & $y=0.0186 x+0.1674$ & 0.4498 \\
\hline & Higuchi & $y=0.1322 x+0.3311$ & 0.8737 \\
\hline & Hixson Crowell & $y=0.4036 x+0.1232$ & 0.4324 \\
\hline & Korsmeyer Peppas & $y=0.9261 x-0.2123, n=0.9261$ & 0.9036 \\
\hline \multirow[t]{5}{*}{$\mathrm{F} 5$} & Zero & $y=0.085 x+0.3160$ & 0.9868 \\
\hline & First & $y=0.0051 x+0.1822$ & 0.2076 \\
\hline & Higuchi & $y=0.6839 x+0.2227$ & 0.8504 \\
\hline & Hixson Crowell & $y=0.2711 x+0.4118$ & 0.5466 \\
\hline & Korsmeyer Peppas & $y=0.4832 x-0.1015, n=0.4832$ & 0.9524 \\
\hline \multirow[t]{5}{*}{ F6 } & Zero & $y=0.7974 x+0.1873$ & 0.8127 \\
\hline & First & $y=0.0072 x+0.184$ & 0.4036 \\
\hline & Higuchi & $y=0.7324 x+0.123$ & 0.6188 \\
\hline & Hixson Crowell & $y=0.7345 x+1.253$ & 0.6127 \\
\hline & Korsmeyer Peppas & $y=0.8761 x-0.044, n=0.8761$ & 0.9907 \\
\hline \multirow[t]{5}{*}{$\mathrm{F} 7$} & Zero & $y=0.119 x+0.3053$ & 0.9303 \\
\hline & First & $y=0.0213 x+0.184$ & 0.3781 \\
\hline & Higuchi & $y=0.7933 x+0.2062$ & 0.8014 \\
\hline & Hixson Crowell & $y=0.1098 x+0.1876$ & 0.5632 \\
\hline & Korsmeyer Peppas & $y=0.5212 x-0.4023, n=0.5212$ & 0.9234 \\
\hline
\end{tabular}




\begin{tabular}{c} 
Table 4: Physicochemical Properties and Permeability Parameters of Piroxicam SLN Gel and Piroxicam Con- \\
ventional Gel (Mean \pm SD; $\boldsymbol{n}=3)$. \\
\hline Formulation
\end{tabular}



Figure 1: TEM Images of Piroxicam Loaded SLNs. (A) Ideal Spherical Shape of Piroxicam SLN (B) SLNs were Smooth, Distinct and Free of Aggregates.

\section{DSC}

The DSC thermogram of pure piroxicam and piroxicam loaded SLN are depicted in Figure 6. The DSC thermogram of pure piroxicam (Figures 6A) showed endothermic peak corresponding to $203.63^{\circ} \mathrm{C}$. This peak is characteristic of melting point of piroxicam. However, the DSC thermogram of piroxicam SLN (Figures 6B) showed an undistinct peak at $53.21^{\circ} \mathrm{C}$ (onset time) and it might be due to the melting of stearic acid which has a melting point of around $54-58^{\circ} \mathrm{C} .{ }^{24}$ Also, the formulation was in a gel, i.e, liquid form already, which might have been a reason for its low melting point. In the DSC thermogram of the SLN formulation, the drug peak was not clearly observed thereby indicating its entrapment within the lipid matrix of the formulation.

\section{Permeation of Piroxicam Loaded SLN Gel and Permeation Data Analysis}

The permeation profile of piroxicam from SLN gel and prepared free drug gel are presented in Figures 7. It depicted the steady increase of piroxicam permeation across the pig ear epidermis with time. As expected, piroxicam SLN gel was found to show better permeation as compared to the conventional gel formulation. Piroxicam SLN gel showed an initial lag phase during permeation. The release of piroxicam from SLN gel

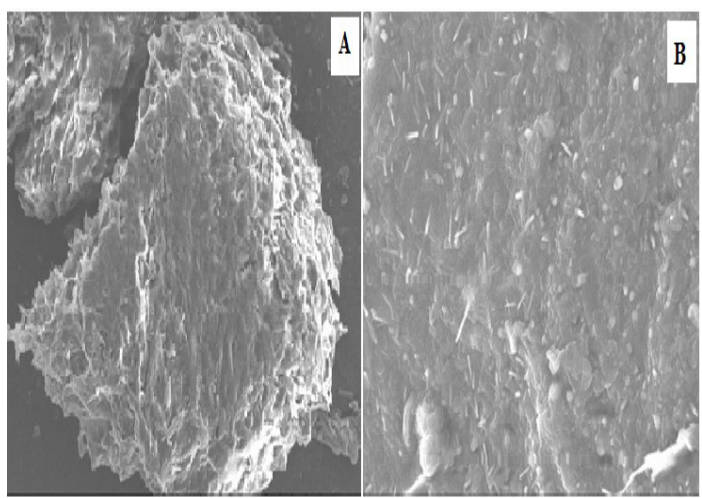

Figure 2: SEM Images of Piroxicam Loaded SLNs. (A) Surface of a smooth, crushed nanoparticle. (B) Presence of distinct SLN particles within the formulation.

occurred in two-phases: initially, piroxicam entrapped within the lipid matrix is unleashed into gel formulations and secondly, piroxicam is released from the gel to the dermal surface. The SLN gel possessed sustained drug release over a period of $24 \mathrm{~h}$. The slower release of the drug from the gel formulation maintained the drug concentration over a extended period of time. The permeability parameters of piroxicam SLN gel and conventional piroxicam gel are given in Table 4. Parameters such as steady-state flux (Jss) and permeability coefficient (Kp) were significantly $(\phi<0.05)$ increased in the SLN gel compared to the conventional gel formulation. The permeability of piroxicam from SLNs was enhanced 1.1 times compared to conventional free drug gel. This indicates that the SLN gel can not only improve the permeation of piroxicam but can also improve its release rate. This indicates the suitability of SLN gel for the topical and/or dermal delivery of piroxicam. The enhancement of permeation and release rate of piroxicam might be the occlusive property of the lipid film formed on the skin surface. They decrease the trans epidermal water loss thereby enhancing piroxicam penetration across the membrane with increased hydration. The effect of surfactant might be another reason for enhancing permeation and release rate of piroxicam through skin. It is 


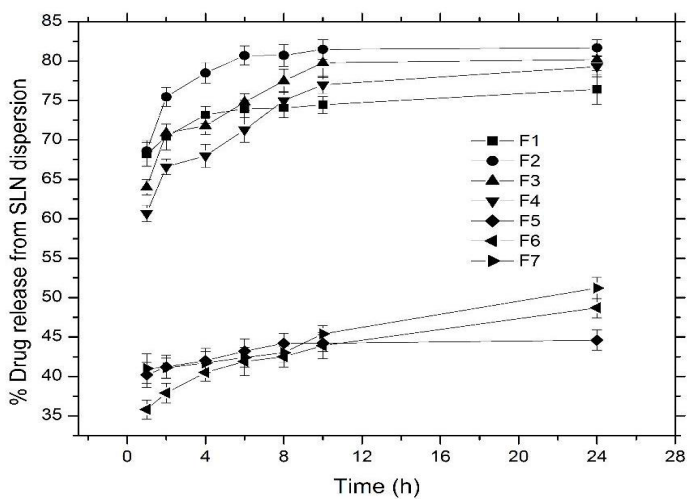

Figure 3: Release Pattern of Piroxicam from Different SLN Formulations (Mean $\pm \mathrm{SD} ; n=3$ ).

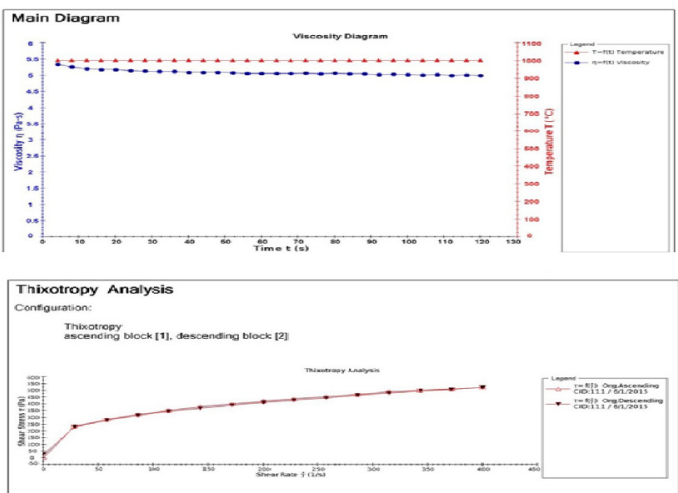

Figure 4: Viscosity and Thixotropy Curve of the F2 SLN Gel. Both these Two Figures Determine the Influence of Time and Temperature on Viscosity of F2 SLN Gel.



Figure 5: Comparative FTIR Spectra of (A) Piroxicam, (B) Stearic acid, (C) Pluronic F68 (D) Phospholipon 90 G, (E) Piroxicam SLN gel.

well known that surfactants have predominant effect on the permeability of drug through biological membranes. The surfactant may permeate into the intracellular regions of stratum corneum and increase their fluidity, eventually solubilizing and extracting the lipid components.



Figure 6: Comparative DSC Thermogram of (A) Piroxicam and (B) Piroxicam SLN Gel.

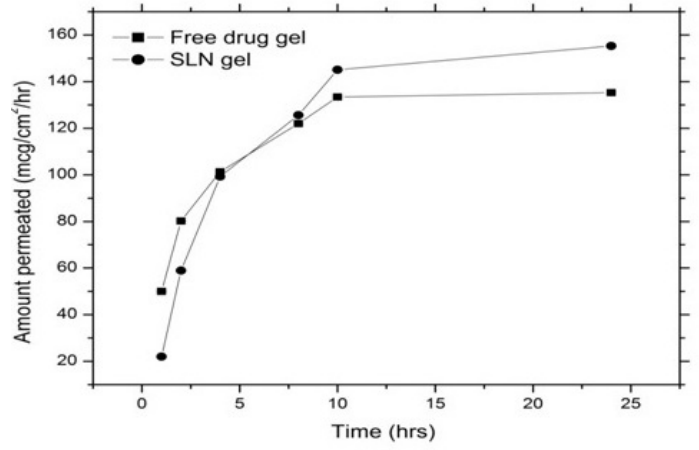

Figure 7: Permeation Profile of Piroxicam from SLN Gel and Conventional Free Drug Gel Across Pig Epidermal Membrane.

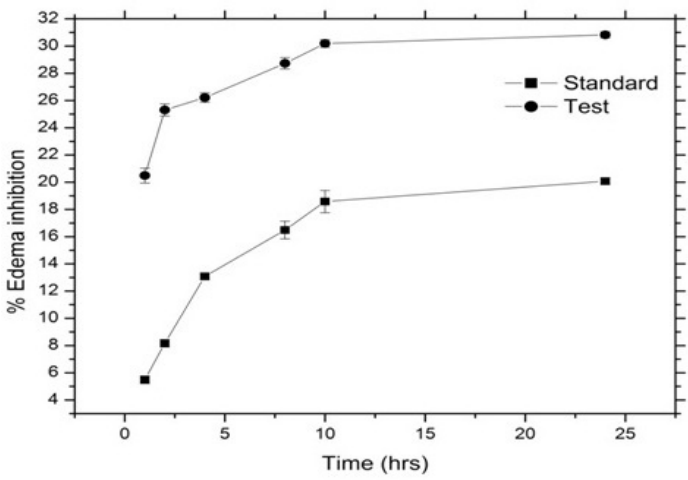

Figure 8: Percentage Edema Inhibition by Piroxicam SLN Gel (Test) Versus Prepared Piroxicam Conventional Gel (Standard) After Topical Application on Wister Albino Rats (Mean \pm $\mathrm{SD} ; n=6$ )

\section{In vivo Efficacy of Piroxicam Loaded SLN Incorporated Gel}

The percentage edema inhibition by piroxicam SLN gel and piroxicam conventional gel with respect to time are presented in Figures 8. The percentage edema inhibition 
by piroxicam conventional gel was $5.49 \pm 0.282 \%$ after $1 \mathrm{hr}$ of topical application. However, percentage edema inhibition after $1 \mathrm{hr}$ on topical application of piroxicam SLN gel was found to be $20.49 \pm 0.543 \%$ After $24 \mathrm{~h}$ of application, the percentage edema inhibition by piroxicam conventional gel and piroxicam SLN gel were found to be $20.08 \pm 0.234 \%$ and $30.82 \pm 0.237 \%$ respectively. This indicates that edema inhibition by piroxicam SLN gel was comparatively more and differed significantly $(p<0.05)$ compared to edema inhibition by piroxicam conventional gel. This might be due to the impressive release pattern of piroxicam from SLN. The fact that piroxicam SLN gel was successful in inhibition of paw edema to an extent greater than that of the piroxicam conventional gel study revealed the applicability and/or effectiveness of the piroxicam SLN gel system for the treatment of arthritic pain and inflammation.

\section{CONCLUSION}

Our study demonstrated the successful preparation of piroxicam incorporated SLNs by high speed homogenization and ultrasonication. The formulated SLNs were subjected to several characteristic evaluations. Evaluation parameters revealed that the percentage of lipid and surfactant have significant $(p<0.05)$ effects on the particle size, drug loading, entrapment efficiency and in vitro release of piroxicam from the SLN formulation. SLN formulation F2 was the most effective formulation with optimum particle size, high entrapment efficiency and improved release profile. The in vitro permeation study indicated revealed sustained permeation of piroxicam from the SLN gel and maintained drug concentration over a prolonged period of time. The permeation parameters indicated the enhancement of piroxicam permeation from SLN gel as compared to conventional gel. Piroxicam loaded SLN formulation was revealed to have local as well as systemic effects as studied by the in vivo anti-inflammatory study. Additionally, topical application of piroxicam SLN gel will be an advantage thereby reducing the gastrointestinal side effects associated with its oral administration. The results depict that the topical application of piroxicam SLN gel system is an effective and safe alternative to the conventional gel and oral formulation for the possible management of inflammatory pain and irritation associated with osteoarthritis, rheumatoid arthritis, edema etc.

\section{ACKNOWLEDGEMENT}

We acknowledge the administrative support obtained from the Department of Pharmaceutical Sciences, Dibrugarh University, Assam, India in carrying out this research work.

\section{FUNDING}

The researchers are grateful to the All India Council for Technical Education for financial support (20/AICTE/ RIFD/RPS(POLICY-1)24/2013-2014).

\section{CONFLICT OF INTEREST}

The authors declare no conflict of interest.

\section{ABBREVIATIONS}

BCS: Biopharmaceutical classification system; DSC: Differential scanning calorimetry; $\mathbf{E}_{\mathrm{r}}$ : Enhancement ratio; DLS: Dynamic light scattering; FTIR: Fourier transform infrared spectra; $\mathbf{J}_{\mathrm{ss}}$ : Steady state flux; $\mathbf{K}_{\mathrm{p}}$ : Permeability coefficient; PDI: Polydispersity index; SEM: Scanning electron microscopy; SLN: Solid lipid nanoparticles; TEM: Transmission electron microscopy.

\section{REFERENCES}

1. Guillory JK. The Merck Index: An Encyclopedia of Chemicals, Drugs and Biologicals Edited by Maryadele J. O'Neil, Patricia E. Heckelman, Cherie B. Koch and Kristin J. Roman. Merck, John Wiley and Sons, Inc., Hoboken, NJ. 2006. xiv+ 2564 pp. $18 \times 26$ cm. ISBN-13 978-0-911910-001. $\$ 125.00$.

2. Brogden RN, Heel RC, Speight TM, Avery GS. Piroxicam: a review of its pharmacological properties and therapeutic efficacy. Drugs. 1981;22(3):16587.

3. Amidon GL, Lennernäs $H$, Shah VP, Crison JR. A theoretical basis for a biopharmaceutic drug classification: the correlation of in vitro drug product dissolution and in vivo bioavailability. Pharm Res. 1995;12(3):413-20.

4. Lands LC, Stanojevic S. Oral non-steroidal anti-inflammatory drug therapy for cystic fibrosis. Cochrane Database Syst Rev. 2007(4):CD001505.

5. Coruzzi G, Venturi N, Spaggiari S. Gastrointestinal safety of novel nonsteroidal antiinflammatory drugs: selective COX-2 inhibitors and beyond. Acta Biomed. 2007;78(2):96-110.

6. Prausnitz MR, Langer R. Transdermal drug delivery. Nat Biotechnol. 2008;26(11):1261-8.

7. Sintov AC, Botner S. Transdermal drug delivery using microemulsion and aqueous systems: influence of skin storage conditions on the in vitro permeability of diclofenac from aqueous vehicle systems. Int J Pharm. 2006;311(1-2):55-62.

8. Kandimalla KK, Kanikkannan N, Singh M. Optimization of a vehicle mixture for the transdermal delivery of melatonin using artificial neural networks and response surface method. J Control Release. 1999;61(1-2):71-82.

9. Khan F, Newton DJ, Smyth EC, Belch JJ. Influence of vehicle resistance on transdermal iontophoretic delivery of acetylcholine and sodium nitroprusside in humans. J Appl Physiol. 2004;97(3):883-7.

10. Drakulic BJ, Juranic IO, Eric S, Zloh M. Role of complexes formation between drugs and penetration enhancers in transdermal delivery. Int $\mathrm{J}$ Pharm. 2008;363(1-2):40-9.

11. Lee $\mathrm{K}$, Lee $\mathrm{CY}$, Jung $\mathrm{H}$. Dissolving microneedles for transdermal drug administration prepared by stepwise controlled drawing of maltose. Biomaterials. 2011;32(11):3134-40.

12. Polat BE, Deen WM, Langer R, Blankschtein D. A physical mechanism to explain the delivery of chemical penetration enhancers into skin during 
transdermal sonophoresis - Insight into the observed synergism. J Control Release. 2012;158(2):250-60.

13. Dhote V, Bhatnagar P, Mishra PK, Mahajan SC, Mishra DK. Iontophoresis: a potential emergence of a transdermal drug delivery system. Sci Pharm. 2012;80(1):1-28.

14. Üner M. Preparation, characterization and physico-chemical properties of Solid lipid nanoparticles (SLN) and Nanostructured lipid carriers (NLC): Their benefits as colloidal drug carrier systems. Pharmazie. 2006;61(5):375-86.

15. Üner M, Yener G. Importance of Solid lipid nanoparticles (SLN) in various administration routes and future perspectives. Int J Nanomed. 2007;2(3):289300.

16. Wissing SA, Lippacher A, Müller $\mathrm{RH}$. Investigations on the occlusive properties of Solid lipid nanoparticles (SLN). J Cosmet Sci. 2001;52(5):31324.

17. Kuo YC, Chen $\mathrm{HH}$. Entrapment and release of saquinavir using novel cationic solid lipid nanoparticles. Int J Pharm. 2009;365(1-2):206-13.

18. Bhaskar K, Anbu J, Ravichandiran V, Venkatesvarlu V, Rao YM. Lipid nanoparticles for transdermal delivery of flurbiprofen: formulation, in vitro, ex vivo and in vivo studies. Lipids Health Dis. 2009;8(1):1-15.
19. Jain SK, Chourasia MK, Masuriha R, Soni V, Jain A, Jain NK, et al. Solid lipid nanoparticles bearing flurbiprofen for transdermal delivery. Drug Deliv. 2005;12(4):207-15.

20. Dasgupta S, Ray S, Dey S, Pal P, Mazumder B. Transdermal lipid nanocarriers: A potential delivery system for lornoxicam. Pharmaceutical Nanotechnology. 2017;5(1):1-15.

21. De Labouret A, Thioune O, Fessi H, Devissaguet JP, Puisieux F. Application of an original process for obtaining colloidal dispersions of some coating polymers. Preparation, characterization, industrial scaling up. Drug Dev Ind Pharm. 1995;21(2):229-41.

22. Das S, Ng WK, Kanaujia P, Kim S, Tan RB. Formulation design, preparation and physicochemical characterizations of solid lipid nanoparticles containing a hydrophobic drug: effects of process variables. Colloids Surf B: Biointerfaces. 2011;88(1):483-9.

23. Hu L, Tang X, Cui F. Solid lipid nanoparticles (SLNs) to improve oral bioavailability of poorly soluble drugs. J Pharm Pharmacol. 2004;56(12):152735.

24. Indian Pharmacopoeia, Ministry of Human Health and Family Welfare, Govt of India, New Delhi. 2006;2.

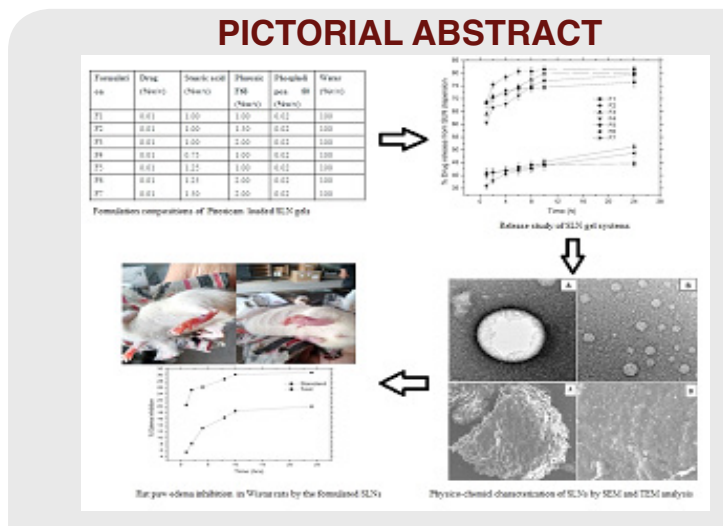

\section{SUMMARY}

- Solid lipid nanoparticles are an emerging drug delivery trend in the recent times. Our experiments have proved the potentiality and appropriateness of piroxicam loaded SLN gel system in treating inflammatory pain and irritation symptoms when delivered topically.

\section{ABOUT AUTHORS}

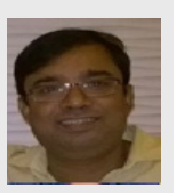

Bhaskar Mazumder is a Professor in the Department of Pharmaceutical Sciences, Dibrugarh University, Assam. His areas of specialization are novel drug delivery system, dosage form design and development etc.

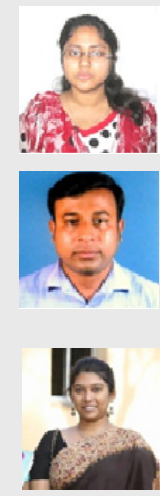

Harshita Krishnatreyya pursued her M. Pharm research work at the Department of Pharmaceutical Sciences, Dibrugarh University in Assam, India. She is currently a registered Ph.D student at the Department of Chemical Technology, University of Calcutta, Kolkata, India.

Sanjay Dey currently works as an Assistant Professor at the Department of Pharmacy in Techno India University, Kolkata, India. He obtained his Ph.D degree from the Department of Pharmaceutical Sciences, Dibrugarh University in Assam, India.

Paulami Pal is a Ph.D research scholar (WOS-A Project, DST) at the Department of Pharmaceutical Sciences, Dibrugarh University in Assam, India.

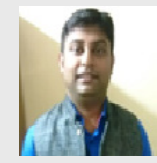

Pranab Jyoti Das, Ph.D from the Department of Pharmaceutical Sciences, Dibrugarh University in Assam, currently works as a Drugs Inspector with the Central Drugs Standard Control, New Delhi, India.

Vipin Kumar Sharma works as an Assistant Professor (Pharmaceutics) in the Department of Pharmaceutical Sciences, Gurukul Kangri Vishwavidyalaya, Haridwar. He was awarded Ph.D degree from the Department of Pharmaceutical Sciences, Dibrugarh University in Assam, India.

Cite this article: Krishnatreyya H, Dey S, Pal P, Das PJ, Sharma VK, Mazumder B. Piroxicam Loaded Solid Lipid Nanoparticles (SLNs): Potential for Topical Delivery. Indian J of Pharmaceutical Education and Research. 2019;53(2S):s82-s92. 